\title{
Recurrent tuberculosis in patients infected with the predominant Mycobacterium tuberculosis outbreak strain in Denmark. New insights gained through whole genome sequencing
}

Folkvardsen, Dorte Bek; Norman, Anders; Rasmussen, Erik Michael; Lillebaek, Troels; Jelsbak, Lars; Andersen, Åse Bengård

Published in:

Infection, Genetics and Evolution

Link to article, DOI:

10.1016/j.meegid.2020.104169

Publication date:

2020

Document Version

Peer reviewed version

Link back to DTU Orbit

Citation (APA):

Folkvardsen, D. B., Norman, A., Rasmussen, E. M., Lillebaek, T., Jelsbak, L., \& Andersen, Å. B. (2020). Recurrent tuberculosis in patients infected with the predominant Mycobacterium tuberculosis outbreak strain in Denmark. New insights gained through whole genome sequencing. Infection, Genetics and Evolution, 80, [104169]. https://doi.org/10.1016/j.meegid.2020.104169

\section{General rights}

Copyright and moral rights for the publications made accessible in the public portal are retained by the authors and/or other copyright owners and it is a condition of accessing publications that users recognise and abide by the legal requirements associated with these rights.

- Users may download and print one copy of any publication from the public portal for the purpose of private study or research.

- You may not further distribute the material or use it for any profit-making activity or commercial gain

- You may freely distribute the URL identifying the publication in the public portal 
First outbreak of multi-drug resistant tuberculosis (MDR-TB) in Denmark involving six Danish-born cases

Camilla Hiul Suppli, Anders Norman, Dorte Bek Folkvardsen, Tina Nørregaard Gissel, Ulla Møller Weinreich, Anders Koch , Christian Wejse, Troels Lillebaek

PII:

DOI: S1201-9712(22)00095-9

Reference: $\quad$ IJID 6008 https://doi.org/10.1016/j.ijid.2022.02.017

To appear in: International Journal of Infectious Diseases

Received date: $\quad 30$ December 2021

Revised date: $\quad 5$ February 2022

Accepted date: 8 February 2022

Please cite this article as: Camilla Hiul Suppli, Anders Norman, Dorte Bek Folkvardsen, Tina Nørregaard Gissel, Ulla Møller Weinreich, Anders Koch, Christian Wejse, Troels Lillebaek, First outbreak of multi-drug resistant tuberculosis (MDR-TB) in Denmark involving six Danish-born cases, International Journal of Infectious Diseases (2022), doi: https://doi.org/10.1016/j.ijid.2022.02.017

This is a PDF file of an article that has undergone enhancements after acceptance, such as the addition of a cover page and metadata, and formatting for readability, but it is not yet the definitive version of record. This version will undergo additional copyediting, typesetting and review before it is published in its final form, but we are providing this version to give early visibility of the article. Please note that, during the production process, errors may be discovered which could affect the content, and all legal disclaimers that apply to the journal pertain.

(C) 2022 Published by Elsevier Ltd on behalf of International Society for Infectious Diseases. This is an open access article under the CC BY-NC-ND license (http://creativecommons.org/licenses/by-nc-nd/4.0/) 
First outbreak of multi-drug resistant tuberculosis (MDR-TB) in Denmark involving six Danish-born cases.

Authors:

Camilla Hiul Suppli, MD, PhD ${ }^{a}$, Anders Norman, MSc PhD ${ }^{a}$, Dorte Bek Folkvardsen MSc PhD ${ }^{a}$, Tina Nørregaard Gissel MDc, Ulla Møller Weinreich, MD, Assoc. Prof. PhD, ${ }^{\mathrm{d}, \mathrm{e}}$ Anders Koch, Prof. PhD, MPH ${ }^{b, f, g}$, Christian Wejse, Assoc. Prof. PhD ${ }^{\text {h,i }}$, Troels Lillebaek Prof. DMSc $\mathrm{a}, \mathrm{b}^{*}$

a. International Reference Laboratory of Mycobacteriology, Statens Serum Institut Artillerivej 5, DK-2300 Copenhagen, Denmark

b. Global Health Section, Department of Public Health, University of Copenhagen Øster Farimagsgade 5, DK-1353 Copenhagen, Denmark

c. Department of Internal Medicine, Region Hospital Viborg, Denmark Heibergs Alle 4F, DK-8800 Viborg, Denmark

d. Department of Respiratory Diseases, Aalborg University Hospital, Aalborg, Denmark Mølleparkvej 4, DK-9000 Aalborg, Denmark

e. Department of Clinical Medicine, Aalborg University, Aalborg, Denmark Søndre Skovvej 15, DK-9000 Aalborg, Denmark

f. Department of Infectious Disease Epidemiology and Prevention, Statens Serum Institut Artillerivej 5, DK-2300 Copenhagen, Denmark

g. Department of Infectious Diseases, Rigshospitalet University Hospital Blegdamsvej 9, DK-2100 Copenhagen, Denmark 
h. GloHAU, Center for Global Health, Dept of Public Health, Aarhus University, Denmark Bartholins Allé 2, DK-8000 Aarhus C, Denmark

i. Dept of Infectious Diseases, Institute for Clinical Medicine, Aarhus University Hospital, Denmark

Palle Juul-Jensens Boulevard 99, DK-8200 Aarhus N, Denmark

*Corresponding author: Professor Troels Lillebaek, International Reference Laboratory of Mycobacteriology, Statens Serum Institut, 5 Artillerivej, DK-2300 Copenhagen, Denmark. tIl@ssi.dk Abstract

Background: Denmark is a tuberculosis (TB) and multi-drug resistant (MDR) TB low-incidence country at 5 and 0.05 cases per 100.000 population, respectively. Until 2018, transmission of MDRTB was nonexistent except for few pairwise related family-cases. In this study we describe the first MDR-TB outbreak in Denmark.

Methods: Based on genotyping of all Mycobacterium tuberculosis (Mtb) culture-positive cases in Denmark spanning three decades, six molecular- and epidemiologically linked Danish-born cases were identified as the first cluster of MDR-TB in Denmark. The primary case was diagnosed posthumously in 2010 followed by five epidemiologically linked cases from 2018 through 2019. Results and conclusion: Through a combination of routine Mtb genotyping and clinical epidemiological surveillance data, we identified the first Danish MDR-TB outbreak spanning 10 years and were able to disclose the specific transmission pathways in detail guiding the outbreak investigations. The occurrence of an MDR-TB outbreak in a resource rich TB low incidence setting like Denmark, highlights the importance of a collaborative control system combining classical contact tracing; timely identification of drug resistant TB through rapid diagnostics; and a close 
collaboration between clinicians, classical- and molecular epidemiologists for the benefit of TB control.

Keywords:

Tuberculosis, TB, MDR-TB, whole genome sequence WGS, contact tracing, Denmark

Introduction

Until the recent advent of COVID-19(Project HOPE, 2020), Mycobacterium tuberculosis (Mtb) remained the leading infectious killer worldwide, with approximately 10 million new cases and 1.3 million deaths per year(World Health Organization, 2020a). In contrast to the steady overall decrease in global TB-incidence, the incidence of multi-drug resistant tuberculosis (MDR-TB) increases by $10 \%$ every year(World Health Organization, $2020 \mathrm{~b}$ ), with the majority of cases observed among previously treated TB-patients, hampering the global TB control-efforts. In 2019, an estimated $3.3 \%$ new TB-cases and $17.7 \%$ of previously treated cases were diagnosed globally with MDR/RR-TB(World Health Organization, 2020a), and in 2016, an estimated 240000 fatal cases were observed following MDR(World Health Organization, 2020b). In the European Union and the European Economic Area, the overall TB-incidence is 10.2 per 100,000 population with an uneven distribution of the MDR-TB cases throughout the region, ranging from $1 \%$ to $20 \%$ (ECDC, 2020). In Eastern Europe, MDR-TB transmission is prevalent(Acosta et al., 2014), whereas MDR-TB is rare in most Western European countries, with the highest prevalence observed among immigrants(Hargreaves et al., 2017). 
Denmark is a TB-low-incidence country with an annual incidence of 5 cases per $100.000^{2}$.

Generally, the prevalence of drug-resistant (DR)-TB is low with isoniazid $(\mathrm{H})$ monoresistance found in around 3\% of cases and approximately three MDR-TB cases per year $(<1 \%)$ (Bang et al., 2010; Statens Serum Institut, 2019). In 2006, the first confirmed transmission of active MDR-TB in Denmark occurred between a foreign-born brother and sister based on identical Mtb genotypes, but until 2018, MDR-TB transmission was nonexistent except for few pairwise related cases within families or friends. (Bang et al., 2010)

In this study, we describe and report the first Danish outbreak of MDR-TB which involves six Danish-born cases over a time period of 10 years, based on genotyping data on all Mtb-culture positive TB cases in the country during the last three decades.

Study population and methods

\section{TB detection and treatment}

In Denmark, TB diagnostics and treatment are free of charge with universal healthcare paid through taxes. All diagnostics are performed in the International Reference Laboratory of Mycobacteriology (IRLM), Statens Serum Institut (SSI), which receives specimens from all patients suspected for TB and other mycobacterioses. Specimens are routinely analyzed by microscopy, PCR and culturing on solid and liquid media. Culture-positive specimens are genotyped and tested for geno- and phenotypic drug susceptibility. The university hospitals in Copenhagen, Odense, Aarhus, and Aalborg handle the clinical management of MDR-TB cases, centrally coordinated by a national MDR-TB council based on WHO recommendations (World Health Organization, 2020c). In Denmark, genotyping is performed by Mycobacterial Interspersed Repeated Units Variable Number of Tandem Repeats analysis (MIRU-VNTR)(Supply et al., 2006), using Bionumerics 
software (v7.6; Applied Maths, Sint-Martens-Latem, Belgium). MIRU-VNTR barcodes are translated into MLVA Mtbc15-9 genotype codes using the MIRU-VNTRplus web service(Weniger et al., 2010) assigned to each culture-positive isolate.

For Whole-Genome Sequencing, the Illumina MiSeq platform is used to generate paired-end ( $2 \mathrm{x}$ $150 \mathrm{bp}$ ) sequencing reads, which are quality trimmed and mapped to the Mycobacterium tuberculosis H37Rv reference genome to identify single-nucleotide polymorphisms (SNPs) as previously described(Folkvardsen et al., 2020; Phelan et al., 2019). In addition, the software tools TB-profiler (v2.8.6) generate a genotypic resistance profile, based on known resistance-mutations, and SpoTyping (v2.1)(Xia et al., 2016) performs in silica spoligotyping for the sequenced isolates. A phylogenetic overview of the cluster based on aligned SNPS, is generated with IQ-TREE software (v1.6.12)(Kalyaanamoorthy et al., 2017; Nguyen et al., 2015) using the built-in automated substitution model selection algorithm and visualized in iTol (v5)(Letunic and Bork, 2019). Mutations conferring resistance to rifampicin and isoniazid are detected by the GenoType MTBDRplus assay, followed by FluoroType MTBDR (both Bruker, Nehren, Germany). Phenotypic drug susceptibility are determined by the proportion method on the BD BACTEC ${ }^{\mathrm{TM}} \mathrm{MGIT}^{\mathrm{TM}} 960$ system (Beckton Dickinson, New Jersey, USA)(Walters and Hanna, 1996).

TB contact tracing, clinical and epidemiological information.

In Denmark, contact tracing is performed for infectious TB-cases to identify other cases of active TB and cases of tuberculosis infection (TBI). Early identification of active TB in contacts reduces disease severity and Mtb transmission, and identification of TBI allows for targeted preventive treatment. Contact tracing is supplemented by results from the nationwide genotyping at the SSI. If specific TB clusters are identified, they are addressed based on the principle of concentric circles 
of exposure(Fox et al., 2013) starting with the closest contacts. The first-line infection control measures include epidemiological interviewing of cases, Quantiferon (Qtf) testing (QuantiFERON-TB Gold PLUS, QIAGEN, Hilden, Germany) and pulmonary x-ray of selected contacts.

Clinical and epidemiological information of the individual cases are reported on a mandatory notification form to the Department of Infectious Disease Epidemiology and Prevention, SSI, which, together with the laboratory, is responsible for TB surveillance in Denmark. In Denmark, TB has been notifiable by law since 1951, with the first mortality records from 1876(Fight Against Tuberc. Denmark, 1950).

\section{Results}

In 2018, three MDR-TB cases with resistance to rifampicin (R) and isoniazid (H) were identified, initially based on rpoB S450L and katG S315T mutations confirmed phenotypically. Two of these cases were sputum smear and Mtb culture-positive and belonged to MIRU-VNTR genotype 122015. The last case was sputum smear-negative, but Mtb culture-positive, and belonged to MIRUVNTR genotype 22299-15, which differs only one locus from 1220-15. Epidemiological contact investigation data showed that all three cases lived in the same apartment building and made regular visits to local pubs.

Case 1 (male, age 47) was admitted in February 2018 due to TB-symptoms over several months and a history of poorly controlled diabetes. He completed 12-month 5-drug combination therapy with good adherence and sputum-negative controls ever since.

Case 2 (male, age 46) was admitted to the department simultaneously with case 1 upon contacting his family physician with symptoms and a history of contact to case 1 . He struggled with multiple 
psychiatric and gastrointestinal disorders and completed the initial treatment partially. He relapsed in Feb 2020 but, by August 2021, he had completed a full 18-month regimen with good adherence. Fortunately, no evolvement of further resistance was seen. 99 people were screened by Qtf test in relation to case 1 and 2, resulting in 11 Qtf positive cases, four people completed 6 months of prophylactic LTBI treatment.

Case 3 (male, age 50) paid a visit to the emergency department in April 2018 and had a chest x-ray taken that showed lesions suspicious for TB, but this was not identified, and he was not admitted. Upon contact tracing, he was identified as a possible MDR-TB case and was admitted to hospital. He struggled with severe alcohol abuse and had low treatment adherence. The patient only completed 9 months of treatment but has had several negative sputum controls subsequently. Case 3 lived in the same apartment building as case 1 and 2 but had been out of the country during the contact investigations of the former two cases. During follow-up of case 3, 13 people were screened by Qtf-test in relation to the case, and all were negative.

By scrutinizing the nationwide genotyping records, a genotypically related TB-case from 2010 (case 4, male age 57) (MIRU-VNTR genotype 1220-15) was identified. After returning from extended backpacking under primitive living conditions in India, this person lived under poor conditions in a closed community in Denmark. At time of admission in 2010, the patient was severely ill and subsequently died from multiple organ failure. Posthumously, the patient was diagnosed with smear-positive MDR-TB with the same genotypic resistance pattern as observed for the three later patients from 2018. Subsequent whole-genome sequencing (WGS) analysis supported the molecular relation between the Mtb strains from the four patients. The exact epidemiological link from case 4 from 2010 (the initial case) to the subsequent three cases from 2018 remains 
unknown, however, case 1 had prior to 2010 regularly bought cannabis from the community where case 4 lived. In 2010, focused x-ray screening of 50 people in the closed community was performed, resulting in 0 extra cases.

Case 5 (male age 44) was admitted to hospital due to liver cirrhosis in the fall of 2018 but was diagnosed with MDR-TB at clinical work-up. This happened six months after the admission of case no. 3. Case 5 had previously had an inconclusive Qtf-test in 2018 in relation to the contact investigation of case 1 but had not received preventive treatment. Case 5 and case 1 lived in the same city. The patient was smear-negative but Mtb culture-positive with the same resistance pattern as cases 1-4 and with MIRU-VNTR genotype 1220-15. The patient died from liver cirrhosis before completion of TB treatment. In relation to this case, 105 people were screened by either Qtf-test or Qtf + chest x-ray, resulting in 1 Qtf-positive case who completed six months of prophylactic LTBI treatment. Simultaneously, 89 people associated with the community where case 4 lived were sputum-smear screened resulting in 0 cases.

In the aftermath of the five cases, in August 2019,48 people were screened by a mobile $x$-ray van specifically targeting frequent visitors to the local pubs as well as the homeless shelters. The screening detected one TB-positive, non-MDR case.

The most recent case (case 6, male age 49) from 2019 was smear and sputum Mtb culture-positive with the same resistance pattern as the former five cases and MIRU-VNTR genotype 1220-15. In 2018, this patient was identified as a contact to cases 1-3 and 5 as he was attending the same pub but had failed to attend the post-exposure examinations. In 2019 , he was admitted to hospital with bronchiectasis, but found to be TB-positive and subsequently completed TB combination 
therapy with sputum negative controls ever since. At contact tracing, 18 people were screened by Qtf test, and all were negative.

Thus, in total, 422 people were screened in the various contact investigations, but apart from the six patients who were found Mtb-positive at hospital admissions, none of the screened persons developed MDR-TB with the MDR-TB outbreak strain, although a few of the screened persons were found Mtb-positive with other strains. Among the six MDR-TB cases, five could be linked epidemiologically to the same apartment building with a pub, whereas the last case had a history of heavy alcohol consumption and was also associated with the pub environment in the same city as cases 1-3. One case (case 4) died before TB diagnosis, one case (case 5) died from cirrhosis before completing treatment, three cases (case 1, 3, 6) completed treatment and one case (case 2) has completed re-treatment for MDR-TB relapse.

\section{Molecular epidemiology}

We used available WGS data from $\approx 4,500$ isolates in the nationwide IRLM strain collection, covering the years 1993 through 2020, to identify possible transmission links to the index case and to explore the immediate genomic neighborhood of the Danish MDR-TB cluster. By including all isolates within 150 SNPs of the index case, we were able to identify 22 additional isolates from 20 TB patients. These included 10 other known isolates in the strain collection (from 9 patients) with MIRU-VNTR 1220-15 genotype, 7 isolates (from 6 patients) with different, but closely related MIRU-VNTR genotypes (19929-15, 7122-15, 22299-15, 1220-28), and 6 isolates without MIRUVNTR types. The related MIRU-VNTR types only differed from 1220-15 at a single locus. None of the isolates had the same $\mathrm{H}$ and $\mathrm{R}$ resistance profile as the Danish MDR-TB cluster, but two isolates (from a single patient) were $\mathrm{H}$ resistant. The average SNP distance between isolates 
belonging to the Danish MDR-TB cluster was 2.5 SNPs (range: 1-6 SNPs) only, while the average distance between members of this cluster to all other isolates was 102 SNPs (range: 74-144 SNPs), ruling out any credible domestic links to case 4 . Furthermore, most of the 22 isolates were collected from foreign-born patients, with countries of origin located in South Asia (Afghanistan, Pakistan, Nepal and India, respectively). We also used the WGS data to construct a phylogenetic tree illustrating the relationships between the 30 isolates (Figure 1). The 30 isolates belong to the East-African-Indian Mycobacterium tuberculosis complex lineage (lineage 3), comprising spoligotypes SIT2145 (the Danish MDR-TB isolates) and SIT26 (all other isolates), which are both members of spoligotype lineage CAS1-Delhi. This particular spoligotype is strongly geographically linked to the particular world region(Couvin et al., 2019), where the index case travelled. Thus, the East-African-Indian origin combined with the short genome distance confirms the relation between the index case and the other cases despite no direct epidemiological link.

\section{Discussion}

In this paper we describe the first MDR-TB outbreak in Denmark. Six Danish-born MDR-TB cases were found to be phylogenetically linked in a cluster of East-African-Indian origin. Based on the molecular epidemiology, we render likely that the case diagnosed in 2010 , hereafter termed the primary case, gave rise to the outbreak spanning the years 2010-2019. The primary case travelled in India prior to being diagnosed with MDR-TB in 2010, whereas the subsequent cases appeared in 2018 through 2019. The long dispersion in time of this outbreak, from primary case to the subsequent cases, highlights the benefits of combining routine molecular epidemiological surveillance data from the laboratory with the classic epidemiological case-based surveillance data from notifications and clinical contact investigations data. 
It is remarkable, that the primary case appeared eight years earlier than the subsequent five cases, and that this link was discovered through laboratory genotyping surveillance. When the subsequent cases were identified, a routine comparison with previously stored Mtb-genotypes identified the likely source of the outbreak. Also, the genotyping results established the recent connections between the outbreak cases and thus supplemented and directed the contact investigations, all factors supporting disease control measures and preventing further spread of MDR-TB.

Four of the six cases in this MDR-TB outbreak were sputum-positive indicating late diagnosis causing more severe disease potentially resulting in additional MDR-TB transmission, challenging TB eradication. The time span of 10 years from the first to the last cases in the outbreak highlights the need for increased focus on screening in risk-group environments to enhance early diagnosis and treatment(Kamper-Jørgensen et al., 2012; Lillebaek et al., 2004). In the Danish outbreak, the primary case had no known connection to any of the other patients, indicating transmission within a public place such as the pub. As seen in this study, although uncommon, transmission in modern housing has occasionally been reported(Dawson et al., 2012; Moreau et al., 2012). In general, Mtb transmission by casual contact is uncommon, but cafes and bars are often poorly ventilated and crowded, and transmission of TB in these public places has been described. In a recent study, Klovdahl et al. demonstrated that such locations often represent the common link between patients with recent TB where a classical epidemiological link could not be established(Klovdahl et al., 2001). This has also been shown in previous studies where molecular methods could not be corroborated by conventional contact tracing(Borrell et al., 2009; Daley et al., 2002; Lillebaek et al., 2001; Maguire et al., 2002). 
The World Health Organization (WHO) recommends systematic screening of risk-groups in TB-lowincidence countries.(World Health Organization, 2013) In these countries, TB tends to be concentrated in urban areas, particularly among high-risk groups such as socially marginalized people(de Vries et al., 2014), as also seen in Denmark(Lillebaek et al., 2013; Stærke et al., 2016). However, TB control among marginalized persons, including screening programs, can be very challenging, calling for TB control interventions based on a multi-sectoral approach targeting the most socioeconomic deprived risk groups in the society using dedicated and trained staff.

Guthrie et al(Guthrie et al., 2018) found that although TB in a low-incidence setting largely arises through reactivation of LTBI in foreign-born persons, local Mtb transmission occurs in discrete populations with distinct disease and risk factor profiles, representing groups for targeted interventions. Active TB-screening in high-risk groups in Denmark has shown that it is feasible to screen such groups using spot sputum culture. This has proven a promising alternative to mobile X-ray screening, identifying $80 \%$ microscopy negative cases coincident with an early, less infectious stage of disease, thereby preventing transmission and increasing disease control in high-risk groups(Jensen et al., 2015). Also, as pointed out by Klovdahl et al(Klovdahl et al., 2001), programs to reduce TB transmission focusing on household members and other close contacts require reconsideration. Modern, multicultural societies encompass different ways of living and different social patterns among groups of people. Therefore, different definitions of close contacts need to be applied for different groups, making contact tracing and TB prevention more efficient. Outbreaks of MDR-TB in countries said to be in the elimination phase of TB, underlines the fact that as long as TB remains a major global health problem, no nation can expect to eliminate the disease(Dahle et al., 2003). 
In the Danish MDR-TB outbreak, none of the cases had received previous TB treatment. However, we know from previous studies that MDR-TB cases are more likely to be re-treatment cases(Faustini et al., 2006; Roberts-Witteveen et al., 2015; Shenoi and Friedland, 2009). As with other bacteria, drug resistance can develop through incomplete, erratic, or inadequate treatment. Based on the genotyping results, the primary case was infected in India, an MDR-TB high-incidence area, and carried the infection back home. Immigration from countries with high rates of MDR-TB is likely to possess an important future challenge for TB control in European countries, although not yet seen often in Denmark(World Health Organization, 2010). In our fellow Nordic countries, MDR-TB remains quite uncommon(ECDC, 2016; Ghebremichael et al., 2008; Vasankari et al., 2012). To our knowledge, Norway is the only Nordic country previously reporting a larger MDR-TB cluster, comprising 20 patients over several years(Dahle et al., 2003). The Norwegian study found the MDR-TB strain to be as transmissible and pathogenic as drug-susceptible strains(Dahle et al., 2003)

Besides the inherent personal risk for patients infected by MDR-TB, this disease entity also comprises a giant strain on health care systems. MDR-TB cases in Europe are reported to have an estimated $9 \%$ increased risk of mortality(ECDC, 2020), are difficult to treat and require many resources both in terms of healthcare workers and finances. The Centers for Disease Control and Prevention (CDC) estimates the cost of one MDR case to be 8 times that of a pan-susceptible case, whereas the number is 15 for an XDR case(Centers for Disease Control and Prevention, 2018). If one imagines that the largest outbreak in Denmark, Cluster 2 (MIRU 1112-15), consisting of more than 1,000 cases and spanning almost 30 years, had been an MDR outbreak; this outbreak would have meant an estimated expense of 60 million Euros (450 million Danish kroner), based on a review of the costs of TB disease in the EU(Diel et al., 2014). This is approximately six times the 
estimated cost for this outbreak of susceptible TB. Emphasizing the importance of diagnosing and stopping disease outbreaks.

This investigation of a drug-resistant TB cluster illustrates the added value of using routine genotyping revealing and delimiting previously unknown routes of transmission. Hereby, analyzing transmission pathways in the Danish setting, high in resources, low in TB-incidence, where TB clusters can be dispersed over a significant amount of years with very few cases. Still, genotyping needs to be combined with classical epidemiological methods to establish the true epidemiological links, as the former cannot stand alone-

\section{Strength and limitations:}

The main strength of our study is the mandatory notification of TB-cases in Denmark leading to high-quality registers. In the Danish Civil Registration System (CRS), all residents in Denmark and persons with residence permits are registered with a unique personal identification number. The use of these numbers allowed us to identify and link individual patients across clinical, epidemiological and laboratory data, allowing complete follow-up. A study of the completeness of tuberculosis (TB) notification systems in six European Union countries, (2014-2016) found the observed and estimated completeness of TB notification in Denmark to be $98.7 \%$ and $98.4 \%$ respectively, indicating a very low proportion of underreporting(Straetemans et al., 2020). In our study, underreporting would mean that we possibly underestimated the size of this cluster and overlooked other clusters. This is not that likely, since all testing and treatment is free of charge, paid via tax in Denmark, and data on treated patients are linked by personal identifier as previously mentioned. Also, MDR-TB patients would likely be difficult to treat with standard 
treatment for susceptible TB and thus, ultimately end up with a microbiological diagnosis and be identified through the automatic mandatory laboratory notification system.

\section{Conclusions:}

This study reports the first Danish outbreak of MDR-TB involving six Danish-born cases over a time-period of 10 years based on clinical-, epidemiological- and genomic routine data. The long timespan between the primary case and subsequent cases illuminates the need for, and advantage of, close collaboration between laboratory-, epidemiological- and clinical services, potentially revealing unknown transmission routes for the benefit of $T B$ control.

Especially in TB low-incidence countries, MDR-TB outbreaks can be difficult to detect at an early stage, as most clinicians will never encounter them. Also, case finding in this socially vulnerable group at risk of TB demands focused efforts by specially dedicated staff. Contact tracing will occur in relation to the occurrence of a clinical case and normally focus on a limited timespan. The addition of molecular genomic typing data can reveal and deliminate previously unknown routes of transmission in low-incidence countries where TB clusters can be dispersed over a significant amount of years.

\section{Funding source}

No funding was received for this work.

\section{Ethical approval}

The study was reported to the Danish Data Protection Agency through the Compliance Division at Statens Serum Institut. The study was purely register-based and did not involve any study participants. For those reasons and according to Danish legislation, scientific ethical approval was not required.

\section{Conflict of interest}

There are no conflicts of interest to declare pertaining to this work. 


\section{References}

Acosta CD, Dadu A, Ramsay A, Dara M. Public Health Action International Union Against Tuberculosis and Lung Disease Health solutions for the poor Drug-resistant tuberculosis in Eastern Europe: challenges and ways forward. PHA 2014;4:2012-4. https://doi.org/10.5588/pha.14.0087.

Bang D, Lillebaek T, Thomsen VO, Andersen ÅB. Scandinavian Journal of Infectious Diseases Multidrug-resistant tuberculosis: Treatment outcome in Denmark 2010;42:288-293. https://doi.org/10.3109/00365540903456282.

Borrell S, Español M, Orcau À, Tudó G, March F, Caylà JA, et al. Factors associated with differences between conventional contact tracing and molecular epidemiology in study of tuberculosis transmission and analysis in the city of barcelona, spain. J Clin Microbiol 2009;47:198-204. https://doi.org/10.1128/JCM.00507-08.

Centers for Disease Control and Prevention. The Costly Burden of DrugResistant TB Disease in the U.S. CDC FACT SHEET 2018. https://www.cdc.gov/nchhstp/newsroom/docs/factsheets/costlyburden-dr-tb-508.pdf (accessed January 28, 2021).

Couvin D, Reynaud Y, Rastogi N. Two tales: Worldwide distribution of Central Asian (CAS) versus ancestral East-African Indian (EAI) lineages of Mycobacterium tuberculosis underlines a remarkable cleavage for phylogeographical, epidemiological and demographical characteristics. PLoS One 2019;14:e0219706. https://doi.org/10.1371/journal.pone.0219706.

Dahle UR, Sandven P, Heldal E, Mannsaaker T, Caugant DA. Deciphering an Outbreak of DrugResistant Mycobacterium tuberculosis. J Clin Microbiol 2003;41:67-72. 
https://doi.org/10.1128/JCM.41.1.67-72.2003.

Daley MF, Steiner JF, Brayden RM, Xu S, Morrison S, Kempe A. Immunization registry-based recall for a new vaccine. Ambul Pediatr 2002;2:438-43. https://doi.org/10.1367/15394409(2002)002<0438:IRBRFA>2.0.CO;2.

Dawson P, Harris TG, Ahuja SD, Anderson JA, Perri BR. Epidemiology Of Tuberculosis Among New Yorkers Living In Public Housing, 2001-2009. Am j Respir Crit Care Med, 2012.

Diel R, Vandeputte J, Vries G de, Stillo J, Wanlin M, Nienhaus A. Costs of tuberculosis disease in the European Union: a systematic analysis and cost calculation. Eur Respir J 2014;43:554-65. https://doi.org/10.1183/09031936.00079413.

ECDC. ECDC Tuberculosis surveillance report 2020 2020:2016-8.

https://www.ecdc.europa.eu/sites/default/files/documents/TB-Surveillancereport_24March2020.pdf.

ECDC. Finland 2016:2016. https://www.euro.who.int/_data/assets/pdf_file/0011/310043/TBsurveillance-report-2016-Finland.pdf?ua=1.

Faustini A, Hall AJ, Perucci CA. Risk factors for multidrug resistant tuberculosis in Europe: A systematic review. Thorax 2006;61:158-63. https://doi.org/10.1136/thx.2005.045963.

Folkvardsen DB, Norman A, Rasmussen EM, Lillebaek T, Jelsbak L, Andersen ÅB. Recurrent tuberculosis in patients infected with the predominant Mycobacterium tuberculosis outbreak strain in Denmark. New insights gained through whole genome sequencing. Infect Genet Evol 2020;80:104169. https://doi.org/10.1016/j.meegid.2020.104169.

Fox GJ, Barry SE, Britton WJ, Marks GB. Contact investigation for tuberculosis: A systematic review 
and meta-analysis. Eur Respir J 2013;41:140-56. https://doi.org/10.1183/09031936.00070812.

Ghebremichael S, Petersson R, Koivula T, Pennhag A, Romanus V, Berggren I, et al. Molecular epidemiology of drug-resistant tuberculosis in Sweden. Microbes Infect 2008;10:699-705. https://doi.org/10.1016/j.micinf.2008.03.006.

Guthrie JL, Kong C, Roth D, Jorgensen D, Rodrigues M, Hoang L, et al. Molecular Epidemiology of Tuberculosis in British Columbia, Canada: A 10-Year Retrospective Study. Clin Infect Dis 2018;66:849-56. https://doi.org/10.1093/cid/cix906.

Hargreaves S, Lönnroth K, Nellums LB, Olaru ID, Nathavitharana RR, Norredam M, et al. Multidrugresistant tuberculosis and migration to Europe. Clin Microbiol infect 2017;23:141-6. https://doi.org/10.1016/j.cmi.2016.09.009.

Jensen SG, Olsen NW, Seersholm N, Lillebaek T, Wilcke T, Pedersen MK, et al. Screening for TB by sputum culture in high-risk groups in Copenhagen, Denmark: A novel and promising approach. Thorax 2015;70:979-83. https://doi.org/10.1136/thoraxjnl-2015-207162.

Kalyaanamoorthy S, Minh BQ, Wong TKF, Von Haeseler A, Jermiin LS. ModelFinder: Fast model selection for accurate phylogenetic estimates. Nat Methods 2017;14:587-9. https://doi.org/10.1038/nmeth.4285.

Kamper-Jørgensen Z, Andersen AB, Kok-Jensen A, Kamper-Jørgensen M, Bygbjerg IC, Andersen PH, et al. Migrant tuberculosis: The extent of transmission in a low burden country. BMC Infect Dis 2012;12:60. https://doi.org/10.1186/1471-2334-12-60.

Klovdahl AS, Graviss EA, Yaganehdoost A, Ross MW, Wanger A, Adams GJ, et al. Networks and tuberculosis: an undetected community outbreak involving public places. Soc Sci Med 
2001;52:681-94. https://doi.org/10.1016/S0277-9536(00)00170-2.

Letunic I, Bork P. Interactive Tree of Life (iTOL) v4: Recent updates and new developments. Nucleic Acids Res 2019;47:W256-9. https://doi.org/10.1093/nar/gkz239.

Lillebaek T, Andersen AB, Bauer J, Dirksen A, Glismann S, De Haas P, et al. Risk of Mycobacterium tuberculosis transmission in a low-incidence country due to immigration from high-incidence areas. J Clin Microbiol 2001;39:855-61. https://doi.org/10.1128/JCM.39.3.855-861.2001.

Lillebaek T, Andersen AB, Rasmussen EM, Kamper-Jorgensen Z, Pedersen MK, Bjorn-Mortensen K, et al. Mycobacterium tuberculosis outbreak strain of Danish origin spreading at worrying rates among Greenland-born persons in Denmark and Greenland. J Clin Microbiol 2013;51:4040-4. https://doi.org/10.1128/JCM.01916-13.

Lillebaek T, Dirksen A, Kok-Jensen A, Andersen ÅB. A dominant Mycobacterium tuberculosis strain emerging in Denmark. Int J Tuberc Lung Dis 2004.

Maguire H, Dale JW, McHugh TD, Butcher PD, Gillespie SH, Costetsos A, et al. Molecular epidemiology of tuberculosis in London 1995-7 showing low rate of active transmission. Thorax 2002;57:617-22. https://doi.org/10.1136/thorax.57.7.617.

Moreau D, Gratrix J, Kunimoto D, Beckon A, Der E, Hansen E, et al. A shelter-associated tuberculosis outbreak: A novel strain introduced through foreign-born populations. Can J Public Heal 2012;103:408-12. https://doi.org/10.1007/bf03405627.

Nguyen L-T, Schmidt HA, von Haeseler A, Minh BQ. IQ-TREE: A Fast and Effective Stochastic Algorithm for Estimating Maximum-Likelihood Phylogenies. Mol Biol Evol 2015;32:268-74. https://doi.org/10.1093/molbev/msu300. 
Phelan JE, O’Sullivan DM, Machado D, Ramos J, Oppong YEA, Campino S, et al. Integrating informatics tools and portable sequencing technology for rapid detection of resistance to antituberculous drugs. Genome Med 2019;11:41. https://doi.org/10.1186/s13073-019-0650-x.

Project HOPE. COVID-19 Is On Track To Become 2020's Leading Infectious Disease Killer, Warns Dr. Tom Kenyon 2020. https://www.projecthope.org/covid-19-is-on-track-to-become-2020s-leadinginfectious-disease-killer-warns-dr-tom-kenyon/11/2020/ (accessed February 16, 2021).

Roberts-Witteveen A, Reinten T, Christensen A, Sintchenko V, Seale P, Lowbridge C. Multidrugresistant tuberculosis in New South Wales, Australia, 1999-2010: A case series report. Int J Tuberc Lung Dis 2015;19:850-6. https://doi.org/10.5588/ijtld.14.0575.

Shenoi S, Friedland G. Extensively drug-resistant tuberculosis: A new face to an old pathogen. Annu Rev Med 2009;60:307-20. https://doi.org/10.1146/annurev.med.60.053107.103955.

Stærke NB, Hilberg O, Wejse C, Hoffmann HJ, Fløe A. Latent tuberculosis infection is prevalent among socially marginalised citizens in Aarhus, Denmark. Dan Med J 2016.

Statens Serum Institut. Tuberculosis- 2018 report on disease occurrence. Epi-News 2019;77.

Straetemans M, Bakker MI, Alba S, Mergenthaler C, Rood E, Andersen PH, et al. Completeness of tuberculosis (TB) notification: inventory studies and capture-recapture analyses, six European Union countries, 2014 to 2016. Eurosurveillance 2020;25:1. https://doi.org/10.2807/15607917.ES.2020.25.12.1900568.

Supply P, Allix C, Lesjean S, Cardoso-Oelemann M, Rüsch-Gerdes S, Willery E, et al. Proposal for standardization of optimized mycobacterial interspersed repetitive unit-variable-number tandem repeat typing of Mycobacterium tuberculosis. J Clin Microbiol 2006;44:4498-510. 
https://doi.org/10.1128/JCM.01392-06.

The Fight Against Tuberculosis in Denmark. vol. 22. Nyt Nordisk Forlag Arnold Busck; 1950.

https://doi.org/10.1109/MPER.2002.4312181.

Vasankari T, Soini H, Liippo K, Ruutu P. MDR-TB in Finland - still rare despite the situation in our neighbouring countries. Clin Respir J 2012;6:35-9. https://doi.org/10.1111/j.1752699X.2011.00242.x.

de Vries G, Aldridge RW, Caylã JA, Haas WH, Sandgren A, van Hest NA, et al. Epidemiology of tuberculosis in big cities of the European union and European economic area countries.

Eurosurveillance 2014;19. https://doi.org/10.2807/1560-7917.ES2014.19.9.20726.

Walters SB, Hanna BA. Testing of Susceptibility of Mycobacterium tuberculosis to Isoniazid and Rifampin by Mycobacterium Growth Indicator Tube Method. J Clin Microbiol 1996;34:1565-7.

Weniger T, Krawczyk J, Supply P, Niemann S, Harmsen D. MIRU-VNTRplus: a web tool for polyphasic genotyping of Mycobacterium tuberculosis complex bacteria. Nucleic Acids Res 2010. https://doi.org/10.1093/nar/gkq351.

World Health Organization. GLOBAL TUBERCULOSIS REPORT 2020 2020a.

http://apps.who.int/bookorders. (accessed November 23, 2020).

World Health Organization. WHO | Drug-resistant TB: global situation. WHO | Drug-Resistant TB Glob Situat 2020b. https://www.who.int/tb/areas-of-work/drug-resistant-tb/global-situation/en/ (accessed November 23, 2020).

World Health Organization. WHO consolidated guidelines on tuberculosis. Module 4, Treatment : drug-resistant tuberculosis treatment. 2020c:98. 
https://www.who.int/publications/i/item/9789240007048 (accessed October 21, 2021).

World Health Organization. Systematic screening for active tuberculosis Principles and recommendations 2013. https://www.who.int/tb/publications/Final_TB_Screening_guidelines.pdf (accessed December 9, 2021).

World Health Organization. Multidrug and extensively drug-resistant TB (M/XDR-TB) 2010 GLOBAL REPORT ON SURVEILLANCE AND RESPONSE 2010. https://apps.who.int/iris/handle/10665/44286 (accessed December 14, 2020).

Xia E, Teo YY, Ong RTH. SpoTyping: Fast and accurate in silico Mycobacterium spoligotyping from sequence reads. Genome Med 2016;8:19. https://doi.org/10.1186/s13073-016-0270-7. 


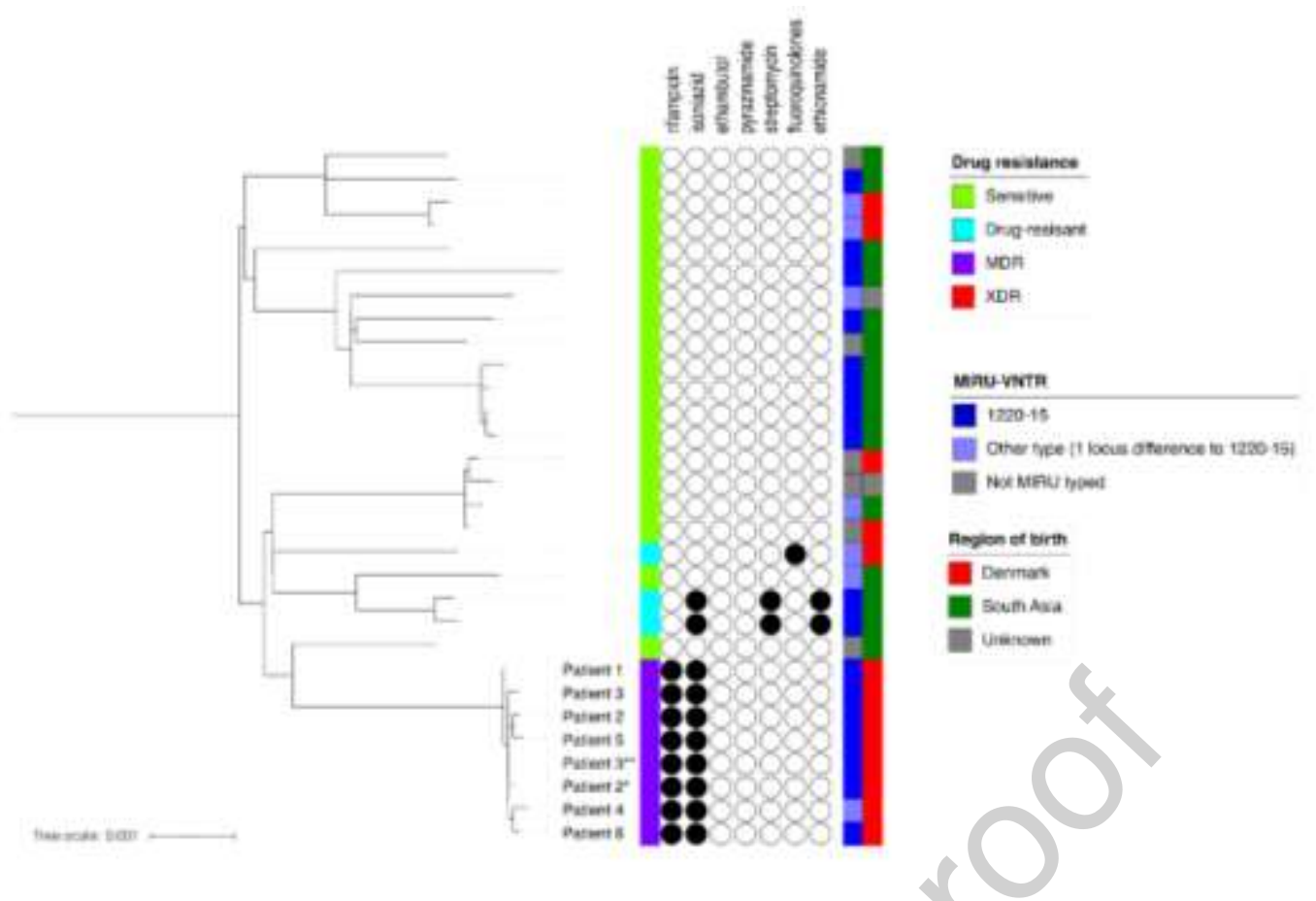

Figure 1. Maximum likelihood tree showing phylogenetic relationships, resistance genotypes, MIRUsubtypes and regions of origin of IRLM strains clustering within 150 SNPs of the MDR-TB cluster (bottom clade). One patient $\left({ }^{*}\right)$ had two sputum samples taken within two days of each other, while another $\left({ }^{* *}\right)$ had recurrent TB after two years. 